\title{
The bacterial microbiota of a parasitic plant and its host
}

\author{
Connor R. Fitzpatrick ${ }^{1,2,3}$, Adam C. Schneider ${ }^{1,2,4}$ \\ ${ }^{1}$ Department of Biology, University of Toronto Mississauga, Mississauga, ON, Canada L5L 1 C6 \\ ${ }^{2}$ Department of Integrative Biology, University of California, Berkeley, California, USA, 94720 \\ ${ }^{3}$ Current address: Department of Biology, University of North Carolina, Chapel Hill, North \\ Carolina, USA, 27599 \\ ${ }^{4}$ Current address: Department of Biology, Hendrix College, Conway, Arkansas, USA, 72032
}

crfitzpat@gmail.com; schneider@hendrix.edu

Corresponding author:

Connor Fitzpatrick

Department of Biology, University of North Carolina

Chapel Hill, North Carolina, USA 27599
(1) 984-215-8934
crfitzpat@gmail.com

Keywords: holoparasite; network analysis; Orobanchaceae; parasitic plant; plant microbiome; Procrustes, microbiome assembly, Orobanche hederae.

Running title: Microbiota of a parasitic plant and its host

Submission: Research paper

Date:

Abstract: 200 words Main body: 4494 words

Figures: 5 (all colour)

Tables: 0

Citations: 60

Supporting Information

Methods: 2425 words

Figures: 11

Tables: 9 


\section{Abstract}

3 How plant-associated microbiota are shaped by, and potentially contribute to the unique

4 ecology and heterotrophic life history of parasitic plants is relatively unknown. Here, we

5 investigate the leaf and root bacterial communities associated with the root holoparasite

6 Orobanche hederae and its host plant Hedera spp. We sequenced the V4 region of the

7 16S rRNA gene from DNA extracted from leaf and root samples of naturally growing

8 populations of Orobanche and infected and uninfected Hedera. Root bacteria inhabiting

9 Orobanche were less diverse, had fewer co-associations, and displayed increased

10 compositional similarity to leaf bacteria relative to Hedera. Overall, Orobanche bacteria

11 exhibited significant congruency with Hedera root bacteria across sites, but not the

12 surrounding soil. Infection had localized and systemic effects on Hedera bacteria, which

13 included effects on the abundance of individual taxa and root network properties.

14 Collectively, our results indicate that the parasitic plant microbiome is derived but distinct

15 from host plant microbiota, exhibits increased homogenization between shoot and root

16 tissues, and displays far fewer co-associations among individual bacterial members.

17 Host plant infection is accompanied by modest changes of associated microbiota at both

18 local and systemic scales compared with uninfected individuals. Our results provide

19 insight into the assembly and function of plant microbiota. 


\section{Introduction}

21 Plants harbour rich assemblages of microorganisms, which vary in diversity and

22 composition across host plant tissues, individuals, and species (Bulgarelli et al., 2013).

23 This variation is driven by innate plant immunity (Lebeis et al., 2015; Stringlis et al.,

24 2018), the quality and quantity of plant-derived resources (Zhalnina et al., 2018), and

25 microbe-microbe interactions (Agler et al., 2016; Durán et al., 2018). Controlled

26 experiments indicate that microbiota may play a role in plant nutrient acquisition

27 (Castrillo et al., 2017) and tolerance to abiotic and biotic stress (Fitzpatrick et al., 2018),

28 including defense from pathogens (Vogel et al., 2016). However, host plant species vary

29 in the composition of their associated microbiota and the causes and ecological

30 consequences of this variation are poorly understood (Fitzpatrick et al., 2018). Increased

31 insight into the assembly and function of plant microbiota requires investigation of plant

32 species that occupy diverse ecological niches (e.g. Angel et al., 2016; Coleman-Derr et

33 al., 2016; Finkel et al., 2016). Such investigation provides an opportunity to address how

34 associated microbiota are shaped by, and potentially contribute to the functional

35 diversity found across plant species.

37 One of the most dramatic niche shifts undergone by plants is the transition from

38 autotrophy to heterotrophy. In heterotrophic plants, resource acquisition from

39 photosynthetic plants occurs indirectly through co-associated mycorrhizal fungi

40 (mycoheterotrophy), or directly through specialized parasitic organs called haustoria.

41 The haustorium attaches to the root or stem vasculature of host plants and acts as a

42 conduit through which material is exchanged primarily from host to parasite 
43 (Sareendenchai and Zidorn 2008; LeBlanc et al., 2012; Spallek et al., 2017).

44 Approximately $2 \%$ of flowering plants directly parasitize other plants to some degree in

45 order to acquire fixed carbon, water, and/or mineral resources. Complete loss of

46 photosynthetic function (i.e. holoparasitism) has evolved at least 13 times in flowering

47 plants alone (Westwood et al., 2010; Braukmann et al., 2013; McNeal et al., 2013).

48 Unsurprisingly, this life-history shift has been accompanied by dramatic ecological and

49 physiological shifts, as well as morphological and genomic changes collectively referred

50 to as 'parasitic reduction syndrome' (Colwell et al., 1994). For example, root

51 holoparasites typically exhibit vestigial, scale-like leaves, loss of a developed root

52 system (Fig. 1A-C), and reduction in chloroplast genome size. However, very few

53 studies have investigated changes induced in the composition and assembly of parasitic

54 plant-associated microbial communities (Kruh et al., 2017) in spite of the mounting

55 evidence that such communities are crucial for plant function and fitness (Vogel et al.,

56 2016; Castrillo et al., 2017; Fitzpatrick et al., 2018).

58 Parasitic plants may also shape the microbiota of their host. Infected host plants have 59 greater demands on their resources because of the significant portion of fixed carbon, 60 water, and nutrients siphoned by the parasitic plant (Fernández-Aparicio et al., 2016).

61 Consequently, infected host plants mount physiological and molecular responses to

62 resist the invading parasite. This can occur over both local scales (i.e. directly

63 parasitized tissue) and systemic, whole plant scales (Hiraoka et al., 2009). These

64 defense responses include the production of reactive oxygen species (Hegenauer et al., 65 2016) and defensive secondary compounds (Serghini et al., 2001; Castillejo et al., 
66 2009), as well as the activation of hormonal pathways governed by jasmonic acid and

67 ethylene (Hiraoka et al., 2009). Plant immunological responses can re-structure

68 associated microbiota due to variation in the susceptibility of microbial taxa to plant

69 immune outputs (Lebeis et al., 2015; Stringlis et al., 2018; Voges et al, 2019). Finally,

70 parasitic plant-derived molecules can alter host root growth (Spallek et al., 2017), which

71 could alter the composition of associated microbiota. Thus, by altering the quality and

72 quantity of plant-derived resources available for microbes, activating innate plant

73 immunity, and altering host plant morphology, parasitic plant infection could perturb the

74 resident microbiota of their hosts.

76 In this study we address three main research questions. First, what are the differences

77 in the diversity, composition, and structure of plant microbiota between a root

78 holoparasite and its autotrophic plant host? We predicted that parasitic plants would

79 exhibit reduced microbial diversity and altered composition and structure relative to host

80 plants potentially due to the 'parasitic reduction syndrome'. Second, does the

81 composition of parasitic plant microbiota track the composition of host plant microbiota?

82 Due to the biased flow of material through the haustorium from host to parasite we

83 predicted that parasitic plant microbial composition would exhibit congruency with that of

84 its host. Third, does infection by a parasitic plant result in perturbations to host plant

85 microbiota? We predicted that parasitic plant infection would alter the diversity and

86 composition of host plant microbiota, possibly a result of induced physiological and

87 immune host responses, which are known to enrich for particular microbial taxa (Lebeis

88 et al., 2015; Stringlis et al., 2018; Voges et al, 2019). To address these questions, we 
89 investigated the plant-associated bacterial microbiota from stable natural populations of

90 Orobanche hederae, the ivy broomrape (hereafter Orobanche), and infected and

91 uninfected individuals of its host plant, Hedera spp., ivy (hereafter Hedera).

92

\section{Materials and Methods}

\section{Study System}

95 Orobanche hederae and various species of Hedera are naturalized on the University of 96 California Berkeley (UCB) campus, but native to Eurasia and North Africa. On the UCB

97 campus, O. hederae was first collected in 2000 and has been persistent at numerous

98 sites for well over 15 years (vouchers at UC and Jepson Herbaria), growing exclusively

99 in near-monocultures of Hedera algeriensis or, rarely, $H$. helix. Curiously, the UCB

100 campus is the only record of $O$. hederae outside its native range with the possible

101 exception of a depauperate specimen collected in 1950 in Raleigh, NC, but not

102 observed subsequently (Musselman, 1980). Similar to other root holoparasites, $O$.

103 hederae seeds germinate in the presence of a suitable host plant and the seedling

104 immediately attaches to a single host root, where it persists for the remainder of its life

105 (Joel et al., 2013). Organs homologous to the leaves and roots of photosynthetic plants

106 are greatly modified in $O$. hederae: scale-like leaves and short, stout hairless roots

107 composed of large, starch-containing cells surrounding the central vascular bundle

108 (Tate, 1925; Joel et al., 2013).

109

110 Plant and soil sampling 
111 We sampled from 4 sites across the UC Berkeley campus (Fig. 1D). At each site we

112 located one infected and one paired but noncontiguous uninfected patch of ivy,

113 assessed by a thorough visual inspection. Infected ivy patches possessed between 20 -

11450 live Orobanche stalks as well as several dozen senesced stalks from previous years,

115 whereas in uninfected patches we were unable to find any living or senesced parasite

116 individuals. Thus, uninfected individuals in our study may represent resistant Hedera

117 genotypes but may also represent individuals that have not been confronted with

118 Orobanche. Infected and uninfected patches are consistent from year to year (A.C.S.,

119 pers. obs.). At each infected patch we carefully excavated flowering Orobanche

120 individuals and the connected host plant root, and followed it until we located a

121 connected leaf (Fig. 1B). The parasite and associated host, including aboveground and

122 belowground organs comprised a single specimen (Fig. 1B). We repeated this process 3

123 times in each infected patch, sampling individuals at least $2 \mathrm{~m}$ apart. For each

124 uninfected patch we collected three ivy individuals at least $2 \mathrm{~m}$ apart, which included

125 aboveground and belowground tissues. Finally, we collected two soil samples

126 (approximately $1 \mathrm{~g}$ sampled from $1-10 \mathrm{~cm}$ soil depth), from each infected and uninfected 127 patch at each site.

$128 \quad$ After collection at each site (approximately 2 hours) plant samples were

129 immediately brought back to the lab in separate bags and prepared for DNA extraction

130 of endophytic microbes using an established protocol (Supporting Information:

131 Methods). From infected patches, we harvested samples from parasite leaves and roots

132 (PL and PR, respectively; Fig. 1C), fully expanded ivy leaves (IL), and two ivy root

133 samples (Fig. 1B): infected roots (IIR; i.e. the specific ivy root being parasitized); and 
134 uninfected roots (IUR; i.e. roots from the same ivy individual and within $50 \mathrm{~cm}$ of the

135 parasite, but not directly being parasitized). From uninfected patches, we harvested ivy

136 leaves and roots (UL and UR, respectively). All samples were standardized by fresh

137 weight and carefully selected to represent homologous organs between the parasite and

138 host plant (full details in Supplementary Data: Methods). Voucher and host information

139 is provided in Table S1.

141 DNA Extraction and Sequencing

142 We used DNeasy PowerSoil extraction kits (Qiagen) following the manufacturer's

143 protocol to isolate DNA from plant tissue and soil samples, then amplified in triplicate,

144 and sequenced the V4 region of the 16S rRNA gene using Illumina MiSeq $2 \times 250 \mathrm{bp}$.

145 Individual fastq files are archived on the NCBI Sequence Read Archive (SRP154488).

146 For a detailed description of PCR and library preparation see Supplementary Data:

147 Methods.

149 Bioinformatics

150 We processed sequences using the R package 'DADA2' v. 1.8.0 (Callahan et al., 2016)

151 (Supplementary Data: Methods). DADA2 infers unique bacterial taxa from amplicon

152 sequence variants (ASVs). We assigned taxonomy to individual ASVs and built a

153 maximum likelihood phylogenetic tree from aligned ASV sequences. Using the R

154 package 'phyloseq' v. 1.24.0 (McMurdie and Holmes, 2012), we removed ASVs that

155 were unassigned to a phylum of Eubacteria (268 ASVs removed), or assigned to plastid

156 and mitochondrial lineages (3268 ASVs removed), which left 6.5 million reads 
157 distributed across 18,329 ASVs. Our final dataset consisted of 103 unique microbiome

158 samples (60 Hedera and 24 Orobanche samples [12 for each of the sample types

159 shown in Fig. 1A], 15 soil samples, and 3 controls), each with on average 63,527 high

160 quality sequences ( \pm 5365 ; standard error).

161

162 Statistical analyses

$163 \alpha$ - and $\beta$-diversity: To test whether $\alpha$-diversity (the number of taxa within a community)

164 and $\beta$-diversity (compositional differences between communities) of leaf and root

165 microbiota varied between Hedera and Orobanche and between infected and uninfected

166 Hedera we used linear mixed models (LMMs; 'Imer' from the R package 'Ime4' v. 1.1-17

167 [Bates et al., 2015]). We calculated a-diversity as ASV richness (R), inverse Simpson's

168 diversity $\left(D^{-1}\right)$, and evenness $\left(D^{-1} / R\right)$. We also calculated phylogenetic diversity (Faith,

169 1992), the sum of the total phylogenetic branch lengths in an assemblage, using the $R$

170 package 'picante' (Kembel et al., 2010). Data for a-diversity indices were In-transformed

171 to meet assumptions of normality and homogeneity of variance. We performed principal

172 coordinates analysis (PCoA) using a weighted UniFrac distance matrix of the ASV

173 dataset (Supplementary Data: Methods).

$174 \quad$ We calculated $\beta$-diversity as the individual sample scores along the first three

175 PCoA axes. Plant species (Hedera spp. or O. hederae), organ type (leaf or root), the

176 interaction between species and organ type, and usable reads were treated as fixed

177 effects, and site and the interaction between species and site were treated as random

178 effects. Using the model object from 'Imer', we tested the significance of fixed effects

179 with type III ANOVA from the R package 'car' v. 3.0-0 using the Kenward-Roger degrees 
180 of freedom approximation (Fox and Weisburg, 2011). To test the significance of random

181 effects we used 'ranova' from the R package 'LmerTest' v. 3.0-1 (Kuznetsova et al.,

182 2015) to perform likelihood ratio tests comparing full and reduced models. Next, we

183 subset the data to include only ivy samples and fit new models to test the effects of

184 infection status on diversity measures. Infection status and usable reads were treated as

185 fixed effects, and site and the interaction between infection status and site were treated 186 as random effects.

188 Differential abundance: We used the raw read counts of ASVs aggregated at each 189 bacterial taxonomic rank to test whether plant species or infection status affected the 190 abundance of individual phyla, classes, orders, families, genera, and ASVs found in 191 leaves and roots (Supplementary Data: Methods). Differential abundance analysis was 192 performed with the R packages 'DESeq2' v. 1.20 .0 (Love et al., 2014) and 'ALDEx2' v. 1931.12 .0 (Fernandes et al., 2013). With each method we tested whether bacterial taxa 194 exhibited differential abundance using selected contrasts: PR vs. IIR; PL vs. IL; IUR vs. 195 IIR; UR vs. IIR; UR vs. IUR; UL vs. IL. The first two contrasts, PR vs. IIR and PL vs. IL, 196 test whether bacterial taxa are differentially abundant between Orobanche and Hedera 197 roots and leaves. The final four contrasts allow us to infer the extent of the effect of 198 Orobanche infection on Hedera microbiota.

200 Network analysis: To understand how differences between Orobanche and Hedera 201 and Hedera infection status influenced bacterial community structure we inferred 202 bacterial co-association networks (Layeghifard et al., 2017) for each of the leaf and root 
203 community types occurring in Orobanche and Hedera (i.e. PL, PR, UL, UR, IL, IR, IIR [n $204=12$ for each type]). We used two methods, which utilize the raw read counts of

205 individual ASVs to infer co-association networks and are designed to be robust to the 206 compositional and sparse nature of microbiome datasets (SparCC [Friedman and Alm, 207 2012]; SPIEC-EASI [Kurtz et al., 2015]). To reduce the bias of anomalous ASVs found 208 at particular sampling sites we included only ASVs found with at least 10 reads in $50 \%$ 209 of samples (Berry and Widder, 2014). We applied this threshold for each community

210 type separately because some ASVs were unique to single community types (e.g. Fig.

211 S3; Table S4) and thus would have been filtered out had the threshold been applied to 212 the entire dataset. This resulted in 7 ASV subsets representing a fraction of the total 213 sequenced reads for each community type (number of ASVs/fraction of total: IIR, 214 229/63\%; IUR, 285/61\%; UR, 289/60\%; PR, 113/51\%; IL, 11/50\%; UL, 10/54\%; PL, $21523 / 56 \%)$.

216

217 The network inference yields a set of bacterial ASVs (nodes) connected by edges, which 218 represent significant co-associations (either positive or negative co-association 219 occurring across samples). To compare networks across sample types we used the $R$ 220 package 'igraph' (Csardi and Nepousz, 2006) to calculate whole network and ASV-level 221 properties thought to be related to individual and community function (Röttjers snd 222 Faust, 2018). For each network, we calculated two measures of individual ASV 223 centrality: degree, the number of edges connected to an ASV; and betweenness 224 centrality, the proportion of the shortest edge paths connecting other members in the 225 network occupied by an ASV (Freeman, 1978). ASVs with high degree number have 
226 numerous co-associations with other network members and may be indicative of hub

227 species (Agler et al., 2015), while ASVs with high betweenness centrality may be

228 mediating interactions between other network members (Röttjers snd Faust, 2018). We

229 used a resampling approach to test whether networks varied in their measures of mean

230 ASV centrality (Supplementary Data: Methods). At the whole network level we

231 calculated edge density and network betweenness centrality. Edge density measures

232 the observed proportion of all possible co-associations among ASVs and network

233 betweenness centrality measures the evenness of betweenness centrality among

234 network members (Freeman, 1978). Values of network betweenness centrality close to

235 zero indicate that all ASVs are equally central, and values close to one indicate a large

236 disparity between the ASVs with the highest and lowest measures of betweenness

237 centrality.

239 Procrustes tests: To test whether Orobanche microbiota track that of their ivy host we

240 performed a series of Procrustes analyses (Peros-Nero and Jackson, 2001)

241 (Supplementary Data: Methods). We used Procrustes analysis to match corresponding

242 sample scores between two community types (i.e. PL, PR, UL, UR, IL, IR, IIR [ $n=12$ for

243 each type]) along the first two coordinate axes (PCoA 1 and PCoA 2) in a PCoA

244 performed on the weighted UniFrac distances among samples. The congruence

245 between two bacterial communities is given by the Procrustes correlation-like statistic

$246\left(t_{0}\right)$, which ranges from 0 (complete discordance) to 1 (perfect congruence). High

247 congruence between two community types (e.g. IIR and PR) indicates that

248 compositional shifts in one community are closely matched by parallel compositional 
249 shifts in the other community (Fig. S7). To understand how individual bacterial taxa may

250 be contributing to the congruence or discordance between parasite and host microbiota

251 we used a leave-one-out approach (Wang et al., 2012). We removed all bacterial ASVs

252 from the parasite dataset classified to a given bacterial phylum, re-calculated weighted

253 UniFrac distances among all samples, obtained sample scores from a new PCoA, and

254 re-calculated $t_{0}$. The effect of excluding a particular bacterial clade on the fit between

255 host and parasite microbiota is given by $\Delta t=\left(t_{\text {excluded }}-t_{0}\right)$. After repeating this for each

256 bacterial phylum we iterated the leave-one-out approach across bacterial orders within

257 phyla whose exclusion lead to large $\Delta t$. We performed the entire leave-one-out

258 approach on root and leaf bacterial communities separately (PR vs. IIR; PL vs. IIR). We

259 used the function 'protest' with 999 permutations from the R package 'vegan' v2.5-2

260 (Oksanen et al., 2018), which calculates $t_{0}$, and performs the permutation test.

261

262 All data and R code used in the analyses are available on figshare (doi: to be 263 determined).

\section{Results}

266 Orobanche root microbiota are less diverse, compositionally dissimilar, and

267 display far fewer co-associations than Hedera

268 Orobanche roots, but not leaves, had reduced a-diversity relative to Hedera (Figs. 2A,

269 S1; Table S2). Both leaf and root bacterial communities of Orobanche were

270 compositionally distinct from those of Hedera (Figs. 2B,C, S2; Table A3: $F_{1,12}=51.32, P$

$271<0.001)$. Across both plant species root communities displayed greater a-diversity than 
272 leaf communities (Figs. 2A, S1; Table S2), and were compositionally distinct (Figs. 2B,C,

273 S2; Table S3: $\left.\mathrm{F}_{1,22}=64.04, P<0.001\right)$.

274 We also found a large number of ASVs unique to Orobanche leaves and roots (Table

275 S4). Orobanche leaf and root communities exhibited greater compositional similarity

276 than those of Hedera (Fig. 2B inset; paired t-test: $\mathrm{t}=-2.52, P=0.01$ ), and shared a

277 greater number of ASVs (Fig. S3). A large number of bacterial taxa across all taxonomic

278 ranks were differentially abundant between Orobanche and Hedera roots and to a lesser

279 extent leaves (Fig. 3, S4; Table S5). Relative to Hedera, Orobanche roots exhibited an

280 increased abundance of the phyla Armatimonadetes, Bacteroidetes, and Proteobacteria,

281 whereas the Planctomycetes were conspicuously absent (Fig. 2C; Table S5). The

282 families Cytophagaceae, Flavobacteriaceae, Phyllobacteriaceae, Rhizobiaceae,

283 Verrucomicrobiaceae were all enriched in Orobanche roots relative to Hedera roots

284 while Haliangiaceae, Micromonosporaceae, Phaselicystidaceae, Polyangiaceae,

285 Rhizomicrobium, Streptomycetaceae were depleted (Table S5). Relative to Hedera

286 leaves, Orobanche leaves had a higher abundance of Acidobacteria, Proteobacteria,

287 and Verrucomicrobia (Fig. 2C; Table S5), including enrichment of members of the well-

288 known plant colonizing bacterial families Enterobacteriaceae, Pseudomonadaceae,

289 Rhizobiaceae.

290

291 In addition to differences in diversity and composition, the structure of root microbiota as

292 measured by bacterial network attributes differed between Orobanche and Hedera

293 (Figs. 4, S5; Table S6). Compared to Hedera, the root bacterial network of Orobanche

294 displayed a near absence of co-associations among ASVs (Fig. 4A-D). This absence 
295 was reflected in the measures of mean ASV centrality, which was approximately ten-fold 296 lower in Orobanche versus Hedera root bacterial networks (Figs. 4F, Kolmogorov-

297 Smirnov test, $D=1, P<0.001$; results are qualitatively similar for degree number). The 298 lack of co-associations in the Orobanche root bacterial network is also evident from the 299 diminished number of associations per bacterial ASV (i.e. degree distribution) in the 300 Orobanche network (Fig. 4E). Additionally, the edge density and network betweenness 301 centrality of the Orobanche root bacterial network was substantially reduced relative to 302 Hedera (Fig. 4A-D), indicating that the reduced number of co-associations in the 303 Orobanche root bacterial network is an attribute shared among all constituent ASVs. In 304 contrast to plant roots, we found few significant associations between members of leaf 305 bacterial communities (Fig. S6; Table S6).

306

307 Orobanche leaf and root microbiota exhibit congruency with Hedera roots

308 Orobanche leaf and root communities significantly resembled the infected (Orobanche

309 leaf: $t_{0}=0.62, P=0.02$; Orobanche root: $t_{0}=0.56, P=0.03$ ) and uninfected roots

310 (Orobanche leaf: $t_{0}=0.61, P=0.02$; Orobanche root: $t_{0}=0.56, P=0.02$ ) of their Hedera

311 hosts (Fig. 5; Table S7). Leaf and root communities within a given species were not

312 congruent (Orobanche: $t_{0}=0.50, P=0.11$; Hedera: $t_{0}=0.33, P=0.53$ ). Hedera root

313 communities were congruent with soil bacterial communities in infected but not

314 uninfected patches (infected patches: $t_{0}=0.91, P=0.04$; uninfected: $t_{0}=0.83, P=0.33$ ).

315 Orobanche roots displayed high but non-significant congruence to soil communities in

316 infected patches $\left(t_{0}=0.95, P=0.08\right)$. 
318 Our leave-one-out approach revealed that a subset of bacterial taxa contribute strongly

319 to either the congruence or discordance between Orobanche and Hedera leaf and root

320 communities (Figs. S7-S10; Tables S8, S9). In root communities, excluding the

321 Burkholderiales (Proteobacteria) led to a large decrease in the Procrustes goodness-of-

322 fit $\left(\Delta t_{0}=-0.133\right)$. By contrast, excluding the Actinomycetales (Actinobacteria) and

323 Flavobacteriales (Bacteroidetes) lead to large increases in the Procrustes goodness-of-

324 fit (Actinomycetales, $\Delta t_{0}=0.043$; Flavobacteriales, $\Delta t_{0}=0.032$ ). The relative

325 abundance of Burkholderiales, but not Actinomycetales, were strongly correlated in

326 Orobanche and Hedera roots, further supporting the Burkholderiales as a strong

327 contributor to congruence (Fig. S9). The fit between Orobanche leaf and Hedera root

328 communities worsened after excluding Pseudomonadales $\left(\Delta t_{0}=-0.112\right)$ and

329 Rhizobiales $\left(\Delta t_{0}=-0.012\right)$, whereas the fit increased after excluding Enterobacteriales

$330\left(\Delta t_{0}=0.032\right)$ and Sphingomonadales $\left(\Delta t_{0}=0.030\right)$, all bacterial orders from the

331 Proteobacteria.

333 Infection status has modest effects on the abundance of individual bacterial taxa

334 and network attributes in Hedera bacterial communities

335 In contrast to our predictions, infection status had no effect on the overall diversity or

336 composition of Hedera leaf and root bacterial communities (Figs. 2A-C; Table S2, S3),

337 although, several ASVs were unique to infected leaves and roots (Table S4). Few

338 bacterial taxa were affected by infection status but these findings appeared to be

339 sensitive to analysis method (Fig. 3, S4; Table S5). Considering only DESeq2 results, a

340 number of taxa did exhibit differential abundance in infected Hedera roots and leaves 
341 consistent with either localized or systemic effects of parasitic plant infection on host

342 plant microbiota (Fig. 3: highlighted taxa). Moreover, our network analyses revealed that

343 infected Hedera root bacterial communities had higher mean ASV and network

344 betweenness centrality than uninfected roots from both infected and uninfected

345 individuals (Fig. 4F; Kolmogorov-Smirnov test: $D=0.45, P<0.001$ ). Thus, infection

346 leads to an increase in the mean but also unevenness in centrality among ASVs

347 associated with Hedera roots.

349 Discussion

350 The bacterial microbiota of a root holoparasite

351 The lower bacterial diversity we found in Orobanche roots parallels their reduced

352 morphological and anatomical structure (Fig. 2A) (Tate, 1925), perhaps due to the

353 availability of fewer niches for microbes. In addition to overall diversity, Orobanche and

354 Hedera significantly differed in the composition of both leaf and root bacteria (Fig. 2B,

355 C). Variation in functional traits (e.g. leaf and root mass per area, leaf nitrogen content)

356 and ecological strategies among plants are thought to contribute to differences in leaf

357 and root microbiota among plant species (Kembel et al., 2014; Laforest-Lapointe et al.,

358 2016; Fitzpatrick et al., 2018). Our results support this paradigm for bacteria composition

359 and root diversity, but foliar bacteria appear less sensitive to a shift to heterotrophy in

360 their host that root-associated bacteria (Fig 2B, Fig. S4, Table S5).

361

362 Like other ecological communities, interactions among microbial species are thought to

363 be an important determinant of the overall composition and function of plant microbiota 
364 (Agler et al., 2016; Durán et al., 2018). Remarkably, we found a near absence of

365 microbial co-associations in the roots of Orobanche, while in Hedera roots we were able

366 to robustly identify numerous co-associations among bacterial taxa (Fig. 4). Shi et al.

367 (2016) proposed that the increased complexity of microbial networks found in

368 rhizosphere versus bulk soil could be due to increased interactions among rhizosphere

369 taxa including microbial cross-feeding, competition and other forms of antagonism.

370 However, niche differences can also result in significant negative and positive co-

371 associations among microbial taxa as environmental variation across habitats, including

372 hosts or host organs, drives species co-occurrence (Zhou et al., 2011; Shi et al., 2016;

373 Freilich et al., 2018). In the context of plant roots, simpler bacterial networks could be

374 the result of fewer persistent microbial interactions and/or available niches across

375 sampled plants. This is not to say that microbial interactions or environmental filtering

376 are absent but rather that they are inconsistent across Orobanche roots, suggesting a

377 greater role for stochastic processes in bacterial community assembly, though this

378 remains to be tested experimentally.

379

380 The generality and functional consequences of the patterns of bacterial diversity and

381 enrichment associated with heterotrophic plants shown here are unknown and require

382 further study using a range of plant parasites and hosts. The Orobanchaceae include

383 the full spectrum of trophic life-histories, including free-living autotrophs, facultative, and

384 obligate parasites such as $O$. hederae. This presents a unique opportunity to test how

385 variation in the degree of parasitism shapes microbial dynamics in both hosts and

386 parasites. Nonetheless, based on the reduced diversity and simpler network structure in 
387 this host-parasite system, we propose that the concept of "parasitic reduction syndrome"

388 (Colwell, 1994) may be expanded to include microbiome reduction as well.

390 Assembly of the parasitic plant microbiota

391 Holoparasitic plants obtain their requisite energy, nutrients, and water from their hosts

392 by way of haustoria, which also allow symplastic and apoplastic transfer of nucleic acids,

393 proteins, and microorganisms (LeBlanc et al., 2012; Spallek et al., 2017). Consequently,

394 the assembly of parasitic plant microbiota is likely shaped by factors associated with

395 host plants (Sheng-Liang et al., 2014; Kruh et al., 2017; Cui et al., 2018). In this study,

396 Orobanche leaf and root bacterial communities displayed compositional shifts congruent

397 with root bacterial communities from infected Hedera (Fig. 5; Table S6). Importantly, we

398 found strong congruency between surrounding soil bacterial composition and the root

399 communities of Hedera, but not Orobanche, indicating that soil environmental features

400 are not solely driving corresponding shifts in parasite and host root microbiota (Fig. 5;

401 Table S6). Instead, the haustorial transfer of microorganisms or plant-derived molecules,

402 which may act to structure microbiota occurring in both Orobanche roots and leaves,

403 could drive this congruence (Kruh et al., 2017). Though we cannot exclude the

404 possibility that Orobanche may be shaping the microbiota of their hosts, we think it

405 unlikely due to the predominantly (albeit not exclusively) one-way flow of haustorial

406 transfer from host to parasite (Serghini et al., 2001). For example, O. hederae can

407 sequester antimicrobial polyacetylenes from ivy hosts (Avato et al., 1996;

408 Sareendenchai and Zidorn, 2008). Orobanche sequestration coupled with host plant

409 variation in the identity or abundance such molecules would lead to congruence in the 
410 composition of associated microbiota between parasite and host. Further study using a

411 holoparasite with a wider host breadth such as Aphyllon purpureum, which parasitizes

412 various members of the Apiaceae, Asteraceae, and Saxifragales (Schneider et al.,

413 2016), could test this hypothesis.

415 Particular bacterial taxa contributed most strongly to our observed congruence or

416 discordance between Orobanche and Hedera microbiota (Figs. S7, S8; Tables S8, S9).

417 Removing the Burkholderiales reduced the congruence between Orobanche and

418 Hedera root microbiota, indicating that their abundance is tightly linked across hosts and

419 parasites (Fig. S8, S9A). In contrast, removing the Actinomycetales increased

420 Orobanche and Hedera root microbiota congruence, indicating that their abundance is

421 decoupled across parasitic plants and hosts during infection (Fig. S9B). The fact that

422 different bacterial taxa contribute to the congruence of either Orobanche leaf or root,

423 and Hedera root microbiota, respectively, (Figs. S7, S8; Tables S8, S9) lends support to

424 our previous finding that the mechanisms structuring parasite leaf and root communities

425 are not entirely overlapping. Though their phylum-level makeup was distinct (Fig. 2C),

426 the leaf and root communities of Orobanche were compositionally similar (Fig. 2B inset).

427 Two non-exclusive explanations for this include increased overlap in the microbial

428 habitats of Orobanche leaves and roots versus Hedera, or perhaps a larger role for

429 stochastic processes governing the assembly of Orobanche microbiota. The reduced

430 complexity found in the Orobanche root bacterial network further supports a diminished

431 role of microbe-microbe interactions and niche-based processes in microbiome

432 assembly. The patterns of Orobanche bacterial diversity and community assembly 
433 exhibit intriguing parallels with the microbiota of eukaryotic parasites of animal hosts

434 (e.g. Husnik, 2018) and suggest that general microbial dynamics may exist in host-

435 parasite systems across plant and animal kingdoms (Dheilly, 2014; Dheilly et al., 2015).

437 The effect of infection status on host plant microbiota

438 Infection by a parasitic plant can induce host plant morphological, physiological, 439 molecular, and transcriptional responses (Westwood et al., 1998; Castillejo et al., 2009;

440 Hiraoka et al., 2009; Hegenauer et al., 2016). In response to infection by the root

441 holoparasite O. cernua, sunflowers synthesize coumarins (Serghini et al., 2001),

442 compounds that are known to inhibit fungal pathogens and also reshape the root

443 microbiome due to antimicrobial effects (Stringlis et al., 2018). In our study, several

444 bacterial taxa exhibited differential abundance consistent with either localized or

445 systemic effects of parasitic plant infection on host microbiota (Fig. 3). The genus

446 Phytohabitans was reduced in only directly infected roots, while Flavobacterium was

447 reduced in both infected and uninfected roots from infected individuals, indicative of

448 systemic effects of infection on Hedera root microbiota. Kruh et al., (2017) found that

449 Flavobacterium was enriched in the post-attachment but pre-inflorescence stage of the

450 parasitic Phelipanche aegyptiaca during infection of tomato plants. Interestingly,

451 Pseudonocardia, was enriched only in infected Hedera leaves, which suggests that

452 microbial perturbations as a result of infection can be localized to tissues not directly

453 infected, potentially linking above and belowground host ecology (Press and Phoenix,

454 2005). Members of the Pseudonocardia are plant endophytes and also act as antifungal

455 mutualists for various species of attine ants (Sen et al., 2009). In spite of these shifts in 
456 taxon abundance, we found that infection status had no effect on the overall diversity or

457 composition of Hedera microbiota. In contrast, Kruh et al., (2017) found that the

458 microbiota of tomato roots parasitized by $P$. aegyptiaca in a greenhouse setting

459 exhibited compositional similarity to that of their parasite when infected, but also were

460 distinct from the roots of uninfected hosts. However, we found that infected Hedera root

461 bacterial networks exhibited elevated network and ASV-level betweenness centrality

462 relative to uninfected Hedera roots (Fig. 4), suggesting that particular ASVs become

463 increasingly co-associated to others in infected roots. Alternatively, under infection, host

464 roots may promote the development of novel microbial niches, which could perturb the

465 co-associations among bacterial taxa resulting in an increase in the co-occurence of a

466 small number of taxa (Shi et al., 2016). Recent work demonstrates that root associated

467 microbiota of host plants may play an important role in mitigating the negative effects of

468 parasitic plant infection (Sui et al., 2018). To mechanistically link infection status and

469 microbiota, future work should characterize the effect of microbial inoculations across

470 resistant and susceptible genotypes during experimentally controlled parasitic plant

471 infections (e.g. Castillejo et al., 2009; Castrillo et al., 2017).

\section{Supplementary Data}

474 Methods Plant sample preparation, molecular methods, and statistical analyses.

475 Fig. S1 Measures of a-diversity across leaf and root samples from Hedera and 476 Orobanche.

477 Fig. S2 Principle coordinate analyses and corresponding scree plots associated with 478 different measures of community dissimilarity.

479 Fig. S3 Shared ASVs among leaf and root communities.

480 Fig. S4 The proportion of bacterial taxa affected by plant species and Hedera infection 481 status.

482 Fig. S5 Root bacterial networks inferred using SparCC. 
483 Fig. S6 Leaf bacterial networks inferred using SPIEC-EASI.

484 Fig. $\mathbf{S 7}$ Procrustes residuals and bacterial community dendrograms.

485 Fig. S8 Leave-one-out Procrustes analysis comparing Orobanche and Hedera root

486 communities.

487 Fig. S9 The relative abundance of bacterial taxa that contribute to the congruence and 488 discordance between Orobanche and Hedera root communities.

489 Fig. S10 Leave-one-out Procrustes analysis comparing Orobanche leaf and Hedera root 490 communities.

491 Fig. S11 Principle coordinate analysis of soil bacterial communities.

492 Table S1 Genome sizes and sampling locations for each Hedera spp. individual.

493 Table S2 Linear mixed effect model results for the analysis of a-diversity.

494 Table S3 Linear mixed effect model results for the analysis of $\beta$-diversity.

495 Table S4 Bacterial ASVs unique to Orobanche and Hedera leaves and roots.

496 Table S5 Full differential abundance testing results.

497 Table S6 Network attributes of bacterial communities in Orobanche and Hedera.

498 Table S7 Results from Procrustes analyses.

499 Table S8 Results from the leave-one-out Procrustes analysis comparing Orobanche and 500 Hedera root communities.

501 Table S9 Results from the leave-one-out Procrustes analysis comparing Orobanche and 502 Hedera root communities.

503

\section{Acknowledgements}

505 We thank Bruce G. Baldwin for hosting us in his lab at UC Berkeley, and Pat Cosgrove

506 for providing lodging and other logistical support during the course of this study. Devin

507 Coleman-Derr generously donated peptide nucleic acids and Marc T. J. Johnson

508 generously donated indexed 16S V4 primers. Shana McDevitt, Denise Schichnes, Dylan

509 Smith, Lydia Smith, and Bridget Wessa also provided laboratory support. We thank Alan

510 Whittemore for measuring Hedera genome size using flow cytometry and providing

511 species determinations. This work was funded by an NSF Doctoral Dissertation

512 Improvement Grant (DEB-1601504) awarded to ACS.

\section{References}

Agler MT, Ruhe J, Kroll S, Morhenn C, Kim ST, Weigel D, Kemen EM. 2016. Microbial hub taxa link host and abiotic factors to plant microbiome variation. PLoS Biology 14, e1002352. 
Angel R, Conrad R, Dvorsky M, Kopecky M, Kotilínek M, Hiiesalu I, Schweingruber F, Doležal J. 2016. The root-associated microbial community of the world's highest growing vascular plants. Microbial Ecology 72, 394-406.

Avato P, Vitali C, Mongelli P, Tava A. 1997. Antimicrobial activity of polyacetylenes from Bellis perennis and their synthetic derivatives. Planta Medica 63, 503-507.

Bates D, Maechler M, Bolker B, Walker S. 2015. Fitting linear mixed-effects models using Ime4. Journal of Statistical Software 67, 1-48.

Berry D, Widder S. 2014. Deciphering microbial interactions and detecting keystone species with co-occurrence networks. Frontiers in Microbiology 5, doi: 10.3389/fmicb.2014.00219

Braukmann T, Kuzmina M, Stefanović S. 2013. Plastid genome evolution across the genus Cuscuta (Convolvulaceae): two clades within subgenus Grammica exhibit extensive gene loss. Journal of Experimental Botany 64, 977-989.

Bulgarelli D, Schlaeppi K, Spaepen S, Ver Loren van Themaat E, Schulze-Lefert P. 2013. Structure and functions of the bacterial microbiota of plants. Annual Review of Plant Biology 64, 807-838.

Callahan BJ, McMurdie PJ, Rosen MJ, Han AW, Johnson AJ, Holmes SP. 2016. DADA2: High-resolution sample inference from Illumina amplicon data. Nature Methods 13, 581-583.

Castillejo MA, Maldonado AM, Dumas-Gaudot E, Fernández-Aparicio M, Susín R, Diego R, Jorrín JV. 2009. Differential expression proteomics to investigate responses and resistance to Orobanche crenata in Medicago truncatula. BMC Genomics 10, 10.1186/1471-2164-10-294.

Castrillo G, Teixeira PJ, Paredes SH, et al. 2017. Root microbiota drive direct integration of phosphate stress and immunity. Nature 543, 513-518.

Coleman-Derr D, Desgarennes D, Fonseca-Garcia C, Gross S, Clingenpeel S, Woyke T, North G, Visel A, Partida-Martinez LP, Tringe SG. 2016. Plant compartment and biogeography affect microbiome composition in cultivated and native Agave species. New Phytologist 209, 798-811.

Colwell AEL. 1994. Genome evolution in a non-photosynthetic plant, Conopholis Americana [Ph.D. dissertation]. St. Louis (MO): Washington University.

Csardi G, Nepusz T. 2006. The igraph software package for complex network research, InterJournal Complex Systems http://igraph.org. 
Cui JL, Vijayakumar V, Zhang G. Partitioning of fungal endophyte assemblages in root-parasitic plant Cynomorium songaricum and its host Nitraria tangutorum. 2018. Frontiers in Microbiology 9, 10.3389/fmicb.2018.00666.

Dheilly NM, Poulin R, Thomas F. 2015. Biological warfare: Microorganisms as drivers of host-parasite interactions. Infection Genetics Evolution 34, 251-259.

Dheilly NM. 2014. Holobiont-holobiont interactions: redefining host-parasite interactions. PLoS Pathogens 10, e1004093.

Durán P, Thiergart T, Garrido-Oter R, Agler M, Kemen E, Schulze-Lefert P, Hacquard S. 2018. Microbial Interkingdom Interactions in Roots Promote Arabidopsis Survival. Cell 175, 973-983.

Faith DP. 1992. Conservation evaluation and phylogenetic diversity. Biological Conservation 61, 1-10.

Fernandes AD, Macklaim JM, Linn TG, Reid G, Gloor GB. 2013. ANOVA-like differential gene expression analysis of single-organism and meta-RNA-seq. PloS One 8, e67019.

Fernández-Aparicio M, Flores F, Rubiales D. 2016. The effect of Orobanche crenata infection severity in faba bean, field pea, and grass pea productivity. Frontiers in Plant Science 7, 10.3389/fpls.2016.01409.

Finkel OM, Delmont TO, Post AF, Belkin S. 2016. Metagenomic signatures of bacterial adaptation to life in the phyllosphere of a salt-secreting desert tree. Applied and Environmental Microbiology 8, 2854-2861.

Fitzpatrick CR, Copeland J, Wang PW, Guttman DS, Kotanen PM, Johnson MTJ. 2018. Assembly and ecological function of the root microbiome across angiosperm plant species. Proceedings of the National Academy of Sciences USA 115, E1157E1165.

Fox J, Weisberg S. 2011. An $\{R\}$ companion to applied regression. Sage Publications Inc.: Thousand Oaks, CA.

Freeman L. 1978. Centrality in social networks conceptual clarification. Social Networks 1, 215-239.

Freilich MA, Wieters E, Broitman BR, Marquet PA, Navarette SA. 2018. Species co occurrence networks: Can they reveal trophic and non - trophic interactions in ecological communities? Ecology 99, 690-699.

Friedman J, Alm EJ. 2012. Inferring correlation networks from genomic survey data. PLoS Computational Biology 8, e1002687. 
Hegenauer V, Fürst U, Kaiser B, Smoker M, Zipfel C, Felix G, Stahl M, Albert M. 2016. Detection of the plant parasite Cuscuta reflexa by a tomato cell surface receptor. Science 353, 478-481.

Hiraoka Y, Ueda H, Sugimoto Y. 2009. Molecular responses of Lotus japonicus to parasitism by the compatible species Orobanche aegyptiaca and the incompatible species Striga hermonthica. Journal of Experimental Botany 60, 641-650.

Husnik F. 2018. Host-symbiont-pathogen interactions in blood-feeding parasites: nutrition, immune cross-talk and gene exchange. Parasitology 145, 1294-1303.

Joel DM, Gressel J, Musselman J, Lytton J. 2013. Parasitic Orobanchaceae: Parasitic mechanisms and control strategies. Springer, New York City.

Kembel SW, Cowan PD, Helmus MR, Cornwell WK, Morlon H, Ackerly DD, Blomberg SP, Webb CO. 2010. Picante: R tools for integrating phylogenies and ecology. Bioinformatics 26, 1463-1464.

Kembel SW, O'Connor TK, Arnold HK, Hubbell SP, Wright SJ, Green JL. 2014. Relationships between phyllosphere bacterial communities and plant functional traits in a neotropical forest. Proceedings of the National Academy of Sciences USA 111, 13715-13720.

Kruh LI, Lahav T, Abu-Nassar J, Achdari G, Salami R, Freilich S, Aly R. 2017. Hostparasite-bacteria triangle: the microbiome of the parasitic weed Phelipanche aegyptiaca and tomato - Solanum lycopersicum (Mill.) as a host. Frontiers in Plant Science 8, 10.3389/fpls.2017.00269.

Kurtz ZD, Müller CL, Miraldi ER, Littman DR, Blaser MJ, Bonneau RA. 2015. Sparse and compositionally robust inference of microbial ecological networks. PLoS Computational Biology 11, e1004226.

Kuznetsova A, Brockhoff PB, Christensen R. 2017. ImerTest package: tests in linear mixed effects models. Journal of Statistical Software 82, 1-26.

Laforest-Lapointe I, Messier C, Kembel S. 2016. Host species identity, site and time drive temperate tree phyllosphere bacterial community structure. Microbiome 4, 10.1186/s40168-016-0174-1.

Layeghifard M, Hwang DM, Guttman DS. 2017. Disentangling interactions in the microbiome: a network perspective. Trends in Microbiology 25, 217-228.

Lebeis SL, Paredes SH, Lundberg DS et al. 2015. Salicylic acid modulates colonization of the root microbiome by specific bacterial taxa. Science 349, 860-864. 
LeBlanc M, Kim G, Westwood JH. 2012. RNA trafficking in parasitic plant systems. Frontiers in Plant Science 3, 10.3389/fpls.2012.00203

Love MI, Huber W, Anders S. 2014. Moderated estimation of fold change and dispersion for RNA-seq data with DESeq2. Genome Biology 15, 31-21.

McMurdie PJ, Holmes S. 2013. phyloseq: an R package for reproducible interactive analysis and graphics of microbiome census data. PLoS ONE 8, e61217-11.

McNeal JR, Bennett JR, Wolfe AD, Mathews S. 2013. Phylogeny and origins of holoparasitism in Orobanchaceae. American journal of botany 100, 971-983.

Musselman L. 1980. The biology of Striga, Orobanche, and other root-parasitic weeds. Annual Review of Phytopathology 18, 463-489.

Oksanen J, Blanchet FG, Friendly M, et al. 2018. vegan: community ecology package. R package version 2.5-2. https://CRAN.R-project.org/package=vegan.

Peres-Neto PR, Jackson DA. 2001. How well do multivariate data sets match? The advantages of a Procrustean superimposition approach over the Mantel test. Oecologia 129, 169-178.

Press MC, Phoenix GK. 2005. Impacts of parasitic plants on natural communities. New Phytologist 166, 737-751.

Röttjers L, Faust K. 2018. From hairballs to hypotheses-biological insights from microbial networks. FEMS Microbiology Reviews 42, 761-780.

Sareendenchai V, Zindorn, C. 2008. Sequestration of polyacetylenes by the parasite Orobanche hederae (Orobanchaceae) from its host Hedera helix (Araliaceae). Biochemical Systematics and Ecology 36, 772-776.

Schneider AC, Colwell AEL, Schneeweis GM, Baldwin BG. 2016. Cryptic hostspecific diversity among western hemisphere broomrapes (Orobanche s.l., Orobanchaceae). Annals of Botany 118, 1101-1111.

Sen R, Ishak HD, Estrada D, Dowd SE, Hong E, Mueller UG. 2009. Generalized antifungal activity and 454-screening of Pseudonocardia and Amycolatopsis bacteria in nests of fungus-growing ants. Proceedings of the National Academy of Sciences USA 106, $17805-17810$.

Serghini K, Pérez de Luque A, Castejón-Muñoz M, García-Torres L, Jorrín JV. 2001. Sunflower (Helianthus annuus L.) response to broomrape (Orobanche cernua Loefl.) parasitism: induced synthesis and excretion of 7-hydroxylated simple coumarins. Journal of Experimental Botany 52, 2227-2234. 
Sheng-Liang Z, Shu-Zhen Y, Zhen-Ying W, Shuang-Lin C. 2014. Endophytic fungi associated with Macrosolen tricolor and its host Camellia oleifera. World Journal of Microbiology and Biotechnology 30, 1775-1784.

Shi S, Nuccio EE, Shi ZJ, He Z, Zhou J, Firestone MK. 2016. The interconnected rhizosphere: High network complexity dominates rhizosphere assemblages. Ecology Letters 19, 926-936.

Spallek T, Melnyk CW, Wakatake T, Zhang J, Sakamoto Y, Kiba T, Yoshida S, Matsunaga S, Sakakibara H, Shirasu K. 2017. Interspecies hormonal control of host root morphology by parasitic plants. Proceedings of the National Academy of Sciences USA 114, 5283-5288.

Stringlis IA, Yu K, Feussner K, de Jonge R, Van Bentum S, Van Verk MC, Berendsen RL, Bakker PAHM, Feussner I, Pieterse CMJ. 2018. MYB72-dependent coumarin exudation shapes root microbiome assembly to promote plant health. Proceedings of the National Academy of Sciences USA 115, E5213-E5222.

Sui XL, Zhang T, Tian YQ, Xue RJ, Li AR. 2018. A neglected alliance in battles against parasitic plants: arbuscular mycorrhizal and rhizobial symbioses alleviate damage to a legume host by root hemiparasitic Pedicularis species. New Phytologist 221, 470-481.

Tate P. 1925. On the anatomy of Orobanche hederae Duby, and its attachment to the host. New Phytologist 5, 284-293.

Vogel C, Bodenhausen N, Gruissem W, Vorholt JA. 2016. The Arabidopsis leaf transcriptome reveals distinct but also overlapping responses to colonization by phyllosphere commensals and pathogen infection with impact on plant health. New Phytologist 212, 192-207.

Voges MJEEE, Bai Y, Schulze-Lefert P, Sattely E. 2019. Plant-derived coumarins shape the composition of an Arabidopsis synthetic root microbiome. Proceedings of the National Academy of Sciences USA 116, 12558-12565.

Wang C, Zöllner S, Rosenberg NA. 2012. A quantitative comparison of the similarity between genes and geography in worldwide human populations. PLoS Genetics 8, e1002886.

Westwood JH, Yoder Jl, Timko MP, dePamphilis CW. 2010. The evolution of parasitism in plants. Trends in Plant Science 15, 227-235.

Westwood JH, Yu X, Foy CL, Cramer CL. 1998. Expression of a defense-related 3hydroxy-3-methylglutaryl CoA reductase gene in response to parasitization by Orobanche spp. Molecular Plant Microbe Interactions 11, 530-536. 
Zhalnina, K, Louie KB, Hao Z, et al. 2018. Dynamic root exudate chemistry and microbial substrate preferences drive patterns in rhizosphere microbial community assembly. Nature Microbiology 3, 470-480.

Zhou J, Deng Y, Luo F, He Z, Yang Y. 2011. Phylogenetic molecular ecological network of soil microbial communities in response to elevated CO2. mBio 2, e0012211 
Figure 1 An overview of the study system and sampling design. (A) We sampled leaves and roots from Orobanche (inflorescence in foreground) and from infected and uninfected Hedera (green plant in background). Note that the abbreviations used here are used throughout the text. (B) We carefully excavated individual Orobanche and its Hedera host to sample leaves and roots from both organisms. For infected Hedera, we distinguished between infected roots (IIR), which were physically attached to the parasite, and uninfected roots (IUR), which did not exhibit direct physical attachment to any parasite. For uninfected Hedera, we sampled leaves and roots in a similar fashion. (C) We sampled sampled leaves (PL) and roots (PR) for Orobanche, which are homolgous but lack the functionality of leaves and roots found in non-parasitic plants. (D) We sampled plants and soil from paired infected and uninfected ivy patches at four sites on the University of California, Berkeley campus. From infected patches we collected Orobanche and Hedera samples and from uninfected patches we collected only Hedera samples.

Figure 2 Diversity and composition of the bacterial communities found in Orobanche and Hedera. (A) Inverse Simpson's diversity across leaves and roots of both plant species ( $\mathrm{n}=12$ for each community type). Roots exhibited nearly 4-fold higher diversity than leaves (Table S2; $\mathrm{F}_{1,21}=17.17, P<0.001$ ) and parasites had reduced root, but not leaf diversity relative to hosts (Table $\mathrm{S} 2 ; \mathrm{F}_{1,5}=5.45, P=0.06$ ). Hedera infection status had no effect on leaf or root diversity (Table S2). (B) Principle Coordinates Analysis of the weighted UniFrac dissimilarity among bacterial communities. Community composition strongly varied between leaves and roots (Table S3; $\mathrm{F}_{1,22}=64.04, P<$ 0.001 ) and plant species (Table S3; $F_{1,13}=51.32, P<0.001$ ). We also found a significant interaction between plant species and organ type (Table S3; $\mathrm{F}_{1,5}=65.50, P<$ $0.001)$, reflecting the larger compositional differences found between leaf and root microbiota for Hedera versus Orobanche $(\mathrm{H}$ versus $\mathrm{O}$, shown in $2 \mathrm{~b}$ inset; paired t-test: $\mathrm{t}$ $=-2.52, P=0.01)$. Hedera infection status had no main effect on the bacterial community composition, though we found a significant interaction between root infection status and sampling site (Table S3; $\chi^{2}=9.12, P=0.003$ ). (C) The relative abundance of the major bacterial phyla found across leaves and roots of both plant species $(n=12$ for each community type).

Figure 3 Differential abundance of bacterial genera across plant species and infection status. We tested whether bacterial genera (labelled according to phylum classification), exhibited differential abundance across five specific contrasts ( $n=12$ for each contrast level). For example, "PR vs. IIR" tested whether bacterial families in Orobanche roots (PR) exhibited enriched or reduced abundance relative to infected Hedera roots (IIR). We used two analytical methods to test for differential abundance, DESeq2 and ALDEx2, and display the overlapping results in darker shades and the results unique to DESeq2 in lighter shades (e.g. both methods found that the Amycolatopsis [Actinobacteria] were reduced in Orobanche roots relative to Hedera, but only DESeq2 found that they were enriched in uninfected versus infected leaves). Contrasts highlighted with arrows represent localized or systemic effects of infection status on Hedera microbiota, and differences between Orobanche and Hedera. We repeated the analysis at all bacterial taxonomic ranks (see Fig. S4 and Table S5 for full results). 
Figure 4 Orobanche and Hedera root bacterial networks inferred by SPIEC-EASI. We inferred the bacterial network of $(A)$ infected roots of infected Hedera, $(B)$ uninfected roots of infected Hedera, $(C)$ roots of uninfected Hedera, and (D) Orobanche roots $(\mathrm{n}=$ 12 for each community type). Node colour and size represent bacterial phylum classification and abundance (centered log-ratio transformed), respectively. Edge colour and width represent sign (green = positive association, red = negative association), and strength of co-association, respectively. At the whole network-level, we found large differences in the edge density and betweenness centrality between Hedera and Orobanche, but not across infected and uninfected Hedera roots (see Table S6), as reflected in the (E) degree distribution (number of associations per node) among community types. (F) We also found large significant differences in mean betweeness centrality of individual taxa among the root bacterial networks of Hedera and Orobanche, as well as infected and uninfected Hedera roots. We tested significance using a series of Kolmogorov-Smirnov tests on the distributions of mean node-level betweenness centrality estimated from 50 nodes sampled with replacement 10,000 times (see Materials and Methods).

Figure 5 Congruence in compositional change across host and parasite leaves and roots. Circle size is proportional to the Procrustes correlation between PCoA ordinations of weighted UniFrac dissimilarity. For example, compositional change among Orobanche leaf bacterial communities $(\mathrm{PL})$ was not correlated $\left(t_{0}=0.5, P>0.05\right)$ with compositional change in Orobanche roots (PR). Instead turnover among PL communities was positively correlated with compositional change among both infected (IIR: $\left.t_{0}=0.62, P<0.05\right)$ and uninfected (IUR: $t_{0}=0.61, P<0.05$ ) roots of infected hosts. See Table S7 for additional comparisons. 


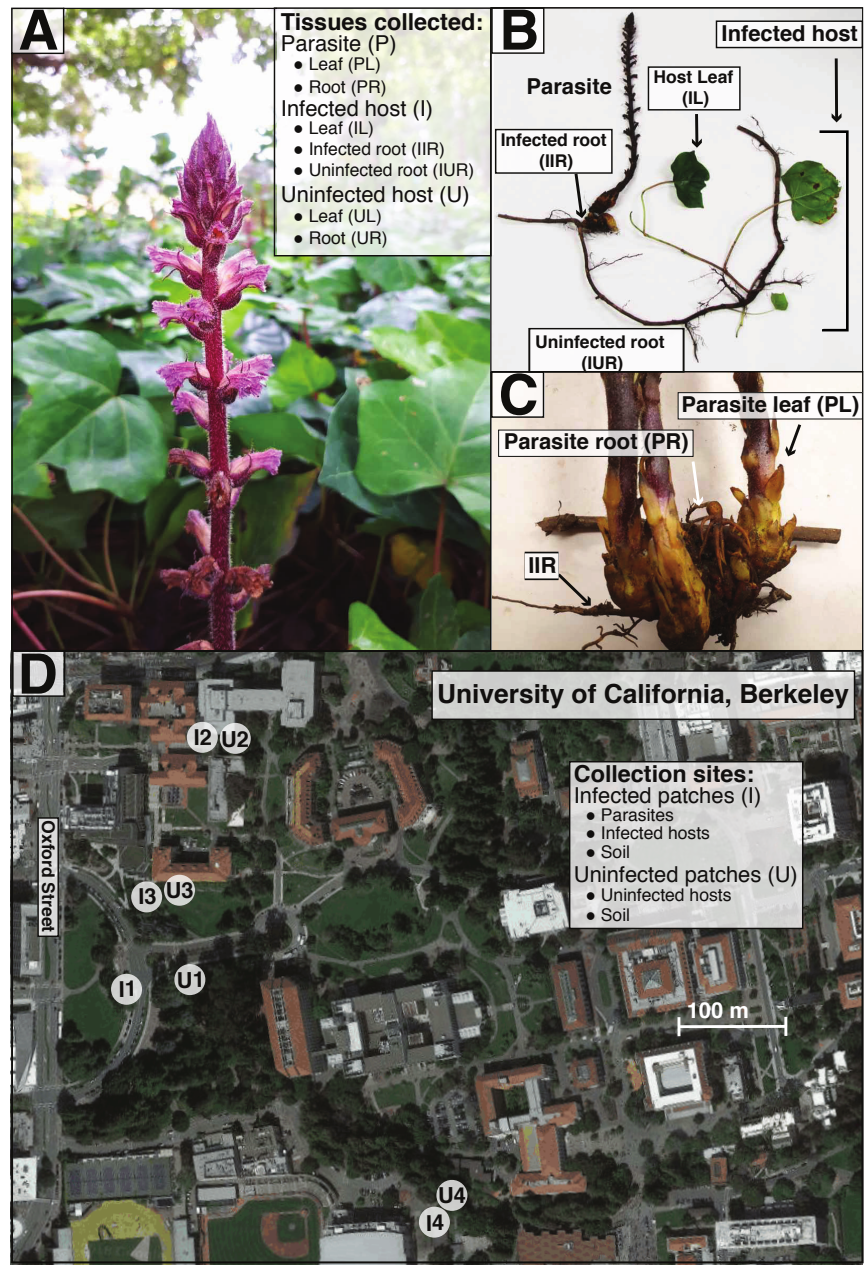

Fig. 1 
Parasite $(\mathrm{P})$

- Leaf (PL)

- Root (PR)

Infected host (I)

- Leaf (IL)

- Infected root (IIR)

- Uninfected root (IUR) Uninfected host (U)

- Leaf (UL)

- Root (UR)

\section{Bacterial phylum}

Actinobacteria

Bacteroidetes

Gemmatimonadetes

Planctomycetes

Verrucomicrobia

Proteobacteria

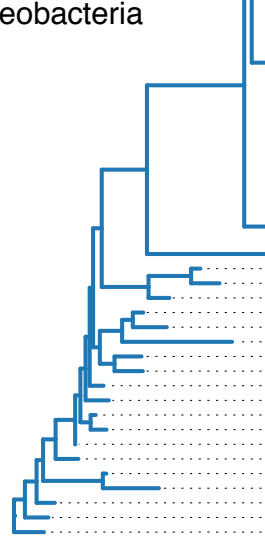

Differential abundance

\section{Contrast}

Fig. 3

$\square$ Enriched (DESeq2 only)

Reduced (DESeq2 only)

Enriched (DESeq2 \& ALDEx2)

Reduced (DESeq2 \& ALDEx2)

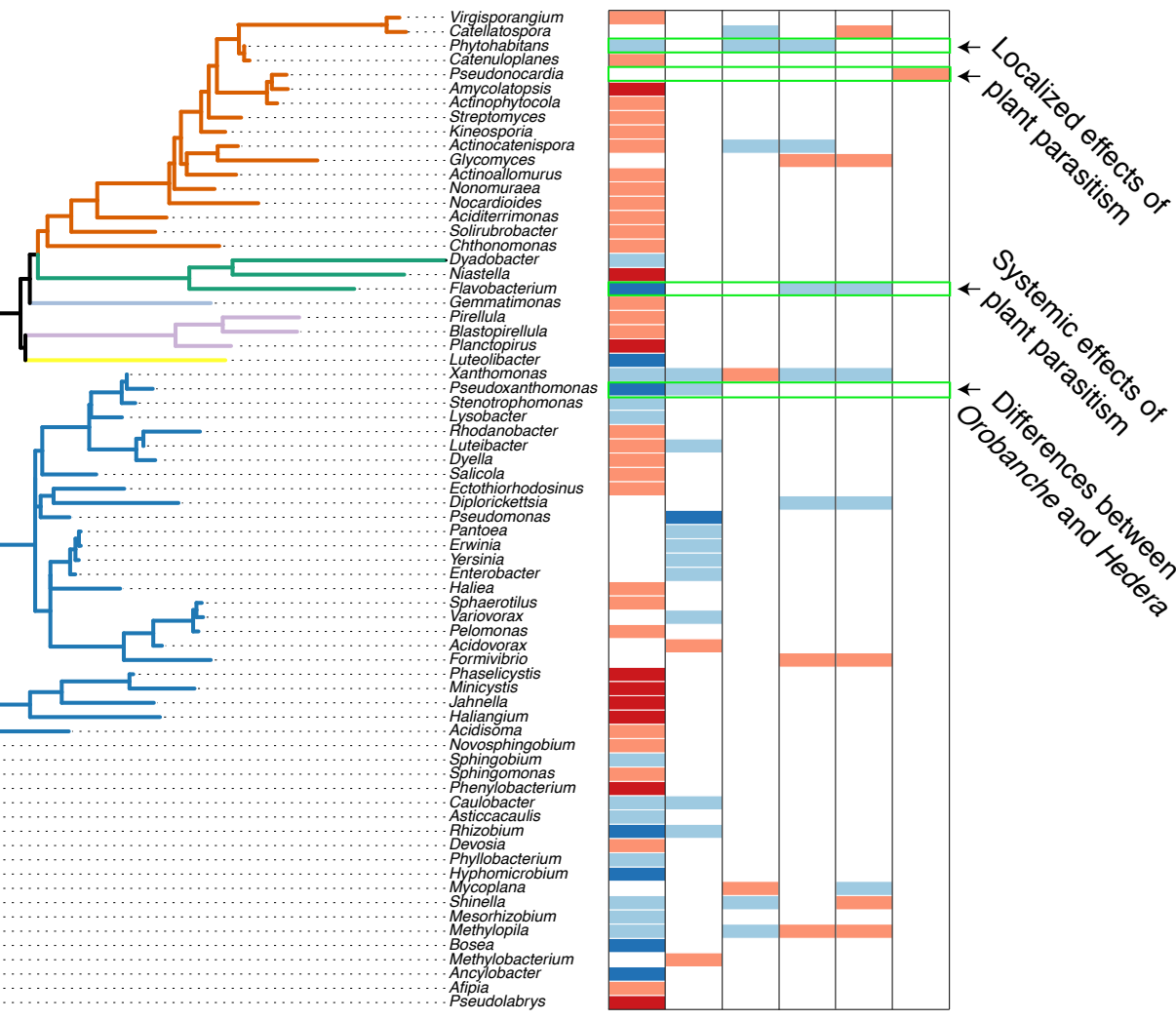


A

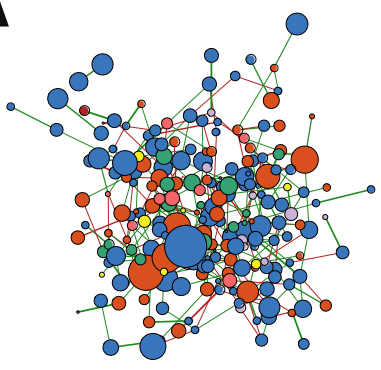

edge density: 0.020 network centrality: 0.035

\section{Bacterial phylum}

Planctomycetes Acidobacteria Verrucomicrobia Bacteroidetes Actinobacteria Proteobacteria

\section{Fig. 4}

B

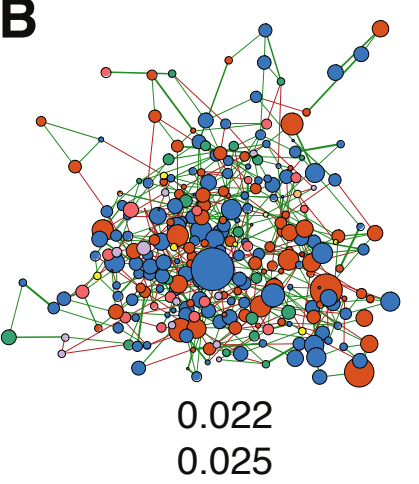

E

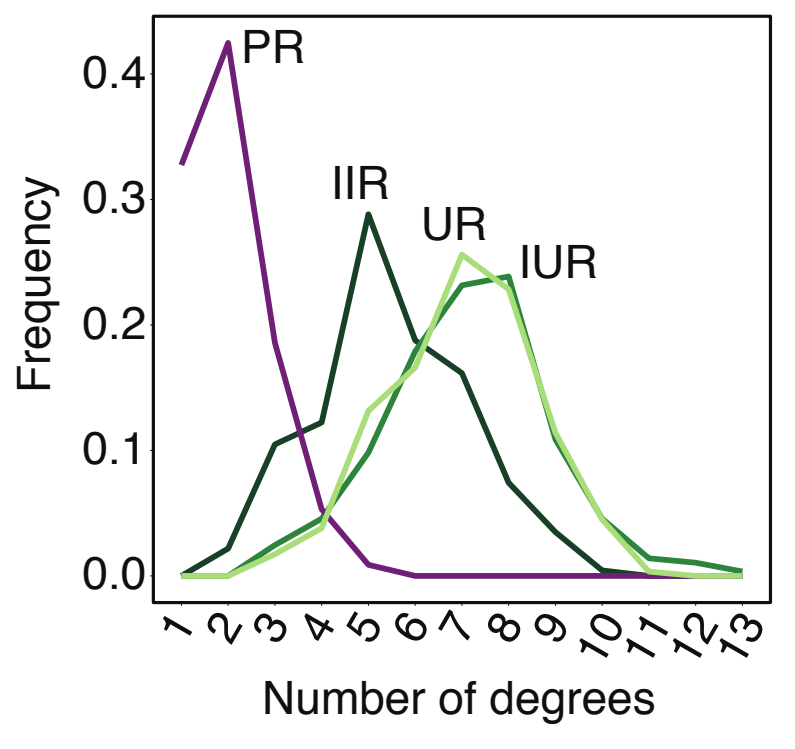

C

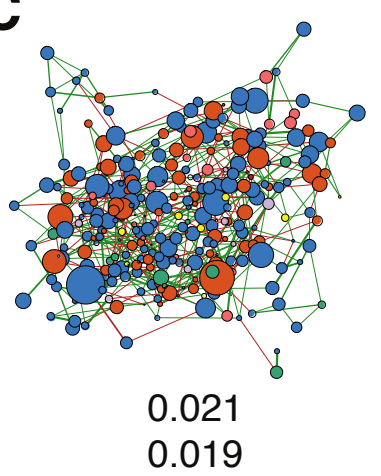

D

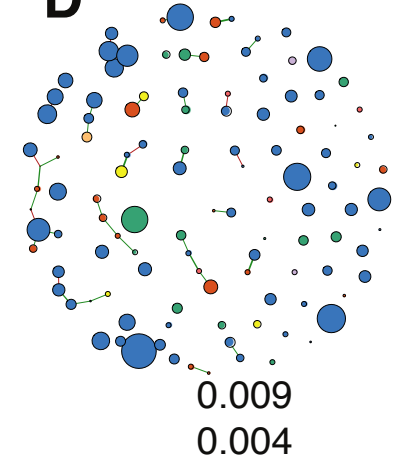

$\mathbf{F}$

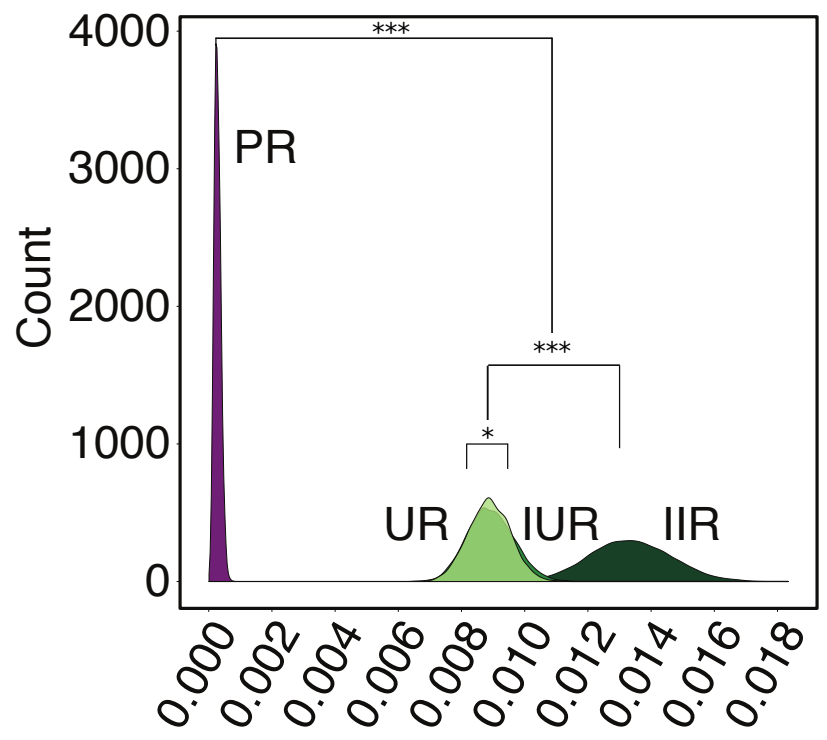

Mean node betweeness centrality 
Significant $(P<0.05)$

$\otimes$ Non-significant

$\mathrm{X}=$ Procrustes correlation $t_{0}$

PL 0.61 IUR

PL 0.62 IIR

PR 0.56IIR

PR 0.56 IUR

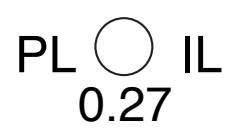

$\mathrm{IL} \bigcirc \mathrm{IIR}$

Parasite $(P)$

- Leaf (PL)

- Root (PR)

Infected host (I)

- Leaf (IL)

- Infected root (IIR)

- Uninfected root (IUR)

Uninfected host (U)

- Leaf (UL)

- Root (UR)

\section{Fig. 5}

